\title{
Aharonov-Bohm Effect, Dirac Monopole, and Bundle Theory
}

\author{
Miguel Socolovsky \\ Instituto de Ciencias Nucleares, Universidad Nacional Autónoma de México \\ Circuito Exterior, Ciudad Universitaria, 04510, México D. F., México \\ Email: socolovs@nucleares.unam.mx
}

\begin{abstract}
We discuss the Aharonov-Bohm $(A-B)$ effect and the Dirac $(D)$ monopole of magnetic charge $g=\frac{1}{2}$ in the context of bundle theory, which allows to exhibit a deep geometric relation between them. If $\xi_{A-B}$ and $\xi_{D}$ are the respective $U(1)$-bundles, we show that $\xi_{A-B}$ is isomorphic to the pull-back of $\xi_{D}$ induced by the inclusion of the corresponding base spaces. The fact that the $A-B$ effect disappears when the magnetic flux in the solenoid equals an integer number of times the quantum of flux associated with the unit of electric charge, reflects here as a consequence of the pull-back of the Dirac connection in $\xi_{D}$ to $\xi_{A-B}$, and the Dirac quantization condition.
\end{abstract}

Keywords: Aharonov-Bohm effect, magnetic monopole, fiber bundles

PACS numbers: 02.40.-k, 02.40.Re, 03.65.Vf, 03.65.-w

\section{Introduction}

As is well known, the Aharonov-Bohm $(A-B)$ effect [1] and the Dirac $(D)$ magnetic monopole [2],[3] proposal have had a profound influence on the development of the gauge theories of fundamental interactions. The first one of these phenomena was immediately verified experimentally [4] and by many others later on [5], while even if Dirac monopoles have not yet being seen in Nature, both grand unified theories [6] and string theories [7] predict their existence.

The description of both the $A-B$ effect and the $D$ monopole are deeply rooted in the concept of gauge potential and therefore in the concept of connection in fiber bundles. The first one provides an explicit evidence of the non-local character of quantum mechanics describing the motion of electrically charged particles in a non-simply connected space [8],[9], while the second one makes unavoidable the use of at least two charts on manifolds to define the gauge potential, leading to the necessity of a description in terms of a non-trivial bundle [10].

The close relationship between both phenomena consists in the facts that when the magnetic flux $\Phi_{A-B}$ is an integer multiple of the quantum of flux $\Phi_{0}=\frac{2 \pi}{|e|}$ associated with the electric charge $|e|$, the $A-B$ effect vanishes, and when $\Phi_{A-B}$ also equals the magnetic flux of the monopole, $\Phi_{D}$, the Dirac quantization condition (D.Q.C.) follows. In this note we want to emphasize this relation at a perhaps deeper level, namely through the relationship between the fiber bundles $\xi_{A-B}$ (trivial) and $\xi_{D}$ (non-trivial) in which both phenomena occur. After some basic material in section 2., in section 3. we exhibit the bundle morphism $\xi_{A-B} \rightarrow \xi_{D}$ induced by the inclusion $\iota$ between the corresponding base spaces, and in section 4. we use $\iota$ to construct the pull-back bundle $\iota^{*}\left(\xi_{D}\right)$, which in turn is proved, in section $\mathbf{5}$., to be isomorphic to $\xi_{A-B}$ i.e.

$$
\xi_{A-B} \cong \iota^{*}\left(\xi_{D}\right) .
$$

This is the main result of the present paper, since it exhibits a deep geometric relation between the $A-B$ effect and the magnetic monopole. Of course, the pull-back of the first Chern class $c_{1}$ of $\xi_{D}, \iota^{*}\left(c_{1}\right)$, vanishes in $\xi_{A-B}$, what is proved in section 6 . In section 7 . we show that the pull-back of the Dirac connection from $\xi_{D}$ to $\xi_{A-B}$ leads to the vanishing of the $A-B$ effect when the D.Q.C. holds, thus setting on purely geometric grounds, one of the basic relations between $A-B$ and $D$. Section $\mathbf{8}$ is devoted to final comments.

We use the natural system of units $\hbar=c=1$. 


\section{Basics}

In Ref. [8], the $U(1)$-bundle associated with the $A-B$ effect [1] with an infinitesimally thin and infinitely long solenoid was shown to be the product-and therefore trivial- bundle

$$
\xi_{A-B}: S^{1} \rightarrow\left(T_{0}^{2}\right)^{*} \stackrel{p r_{1}}{\longrightarrow}\left(D_{0}^{2}\right)^{*}
$$

where $S^{1}=U(1)=\{z \in \mathbb{C},|z|=1\}$ is the structure group, $\left(D_{0}^{2}\right)^{*}$ is the punctured open disk in two dimensions, $\left(T_{0}^{2}\right)^{*}=\left(D_{0}^{2}\right)^{*} \times S^{1}$ is the open solid 2-torus minus a circle, and $p r_{1}$ is the projection in the first entry. One has the homeomorphisms $\left(D_{0}^{2}\right)^{*} \cong\left(\mathbb{R}^{2}\right)^{*}=\mathbb{R}^{2} \backslash\{0\} \cong \mathbb{C}^{*}=\mathbb{C} \backslash\{0\}$. The reason for (2) is that, in the above conditions, by symmetry reasons the space available to the electrically charged particles ("electrons") moving around the solenoid is $\left(\mathbb{R}^{2}\right)^{*}$ which is of the same homotopy type as the circle $S^{1}$. Then the set of isomorphism classes of $U(1)$-bundles over $\left(\mathbb{R}^{2}\right)^{*}$ consists of only one element [11]: the class of the product (trivial) bundle $\left(T_{0}^{2}\right)^{*}$.

On the other hand, the fiber bundles associated with Dirac monopoles [2],[3] of magnetic charge $g=\# k$ with $k$ an integer and \# a number depending on units, are the Hopf bundles [10],[12]

$$
\xi_{D}^{(k)}: S^{1} \rightarrow P_{k}^{3} \stackrel{\pi_{k}}{\longrightarrow} S^{2}
$$

where $P_{0}^{3}=S^{2} \times S^{1}$ (the trivial bundle), $P_{k}^{3} \cong P_{-k}^{3}, S^{2}$ is the 2 -sphere with $S^{2} \cong \mathbb{R}^{2} \cup\{\infty\} \cong \mathbb{C} \cup\{\infty\}$. In particular, we are interested in the case $k=1$ for which $P_{1}^{3} \cong S^{3}$ : the 3 -sphere given by

$$
S^{3}=\left\{\left(z_{1}, z_{2}\right) \in \mathbb{C}^{2},\left|z_{1}\right|^{2}+\left|z_{2}\right|^{2}=1\right\},
$$

$\pi_{3} \equiv \pi$ is the Hopf map [13]

$$
\pi: S^{3} \rightarrow S^{2},\left(z_{1}, z_{2}\right) \mapsto \pi\left(z_{1}, z_{2}\right)=\left\{\begin{array}{c}
z_{1} / z_{2}, z_{2} \neq 0 \\
\infty, z_{2}=0
\end{array}\right.
$$

We denote this non-trivial bundle $\xi_{D}$ :

$$
\xi_{D}^{(1)} \equiv \xi_{D}: S^{1} \rightarrow S^{3} \stackrel{\pi}{\longrightarrow} S^{2} .
$$

The global connection on $\xi_{D}$ corresponding to $g=\frac{1}{2}\left(\#=\frac{1}{2}\right.$ and $\left.k=1\right)$ is the 1 -form $\omega \in \Omega^{1} S^{3} \otimes u(1)$, with $u(1)=\operatorname{Lie}(U(1))=i \mathbb{R}$, given by $[14]$

$$
\omega=\frac{i}{2}(d \chi+\cos \theta d \varphi)
$$

where $\chi, \theta$ and $\varphi$ are the Euler angles in $S^{2}$ or $\mathbb{R}^{3}(\theta \in[0, \pi]$ and $\chi, \varphi \in[0,2 \pi))$. The differential of $\omega$ is the 2 -form

$$
d \omega=-\frac{i}{2} \sin \theta d \theta \wedge d \varphi=-F \in \Omega^{2} S^{3} \otimes u(1)
$$

where $F$ is the field strength

$$
F=i|\boldsymbol{B}| \sin \theta d \theta \wedge d \varphi
$$

with

$$
\boldsymbol{B}=\left(\frac{1}{2}\right) \frac{\boldsymbol{r}}{r^{3}}
$$

the magnetic field of the monopole in $\mathbb{R}^{3} \backslash\{0\}$ (see below).

$\omega$ can be read from the squared length element on $S^{3}$ :

$$
d l_{S^{3}}^{2}(\chi, \theta, \varphi)=\frac{1}{4}\left(d \theta^{2}+\sin ^{2} \theta d \varphi^{2}+(d \chi+\cos \theta d \varphi)^{2}\right)
$$

which, in turn, can be obtained from the identification of $S^{3}$ with the group $S U(2)$ with elements

$$
\left(\begin{array}{cc}
z_{1} & z_{2} \\
-\bar{z}_{2} & \bar{z}_{1}
\end{array}\right)=\left(\begin{array}{cc}
e^{\frac{i}{2}(\varphi+\chi)} \cos \frac{\theta}{2} & e^{\frac{i}{2}(\varphi-\chi)} \sin \frac{\theta}{2} \\
-e^{-\frac{i}{2}(\varphi-\chi)} \sin \frac{\theta}{2} & e^{-\frac{i}{2}(\varphi+\chi)} \cos \frac{\theta}{2}
\end{array}\right)
$$


Covering $S^{2}$ with the open sets $U_{+}$and $U_{-}$respectively defined by $\theta \in[0, \pi)$ (the south pole $S$ excluded) and $\theta \in(0, \pi]$ (the north pole $N$ excluded), considering the pull-back of $\omega$ to $S^{2} \backslash\{N, S\}$ with the local sections

$$
\begin{gathered}
s_{N}: U_{+} \backslash\{N\} \rightarrow S^{3}, s_{N}(\hat{n})=\left(\cos \frac{\theta}{2}, \sin \frac{\theta}{2} e^{i \varphi}\right), \\
s_{S}: U_{-} \backslash\{S\} \rightarrow S^{3}, s_{S}(\hat{n})=\left(\cos \frac{\theta}{2} e^{i \varphi}, \sin \frac{\theta}{2}\right),
\end{gathered}
$$

with $\hat{n}=(\sin \theta \cos \varphi, \sin \theta \sin \varphi, \cos \theta)$, using the inclusion

$$
\begin{gathered}
j: S^{3} \rightarrow \mathbb{R}^{4}, j\left(z_{1}, z_{2}\right)=\left(x_{1}, x_{2}, x_{3}, x_{4}\right) \\
=\left(\cos \left(\frac{\varphi+\chi}{2}\right) \cos \frac{\theta}{2}, \sin \left(\frac{\varphi+\chi}{2}\right) \cos \frac{\theta}{2}, \cos \left(\frac{\varphi-\chi}{2}\right) \sin \frac{\theta}{2}, \sin \left(\frac{\varphi-\chi}{2}\right) \sin \frac{\theta}{2}\right),
\end{gathered}
$$

and defining the 1 -form $\tilde{\omega} \in \Omega^{1} \mathbb{R}^{4} \otimes u(1)$ through

$$
\tilde{\omega}=i\left(x^{1} d x^{2}-x^{2} d x^{1}-x^{3} d x^{4}+x^{4} d x^{3}\right),
$$

one can prove that $j^{*}(\tilde{\omega})=\omega$ and that $s_{N, S}^{*}(\omega)$ are the usual local 1 -forms $A_{ \pm}$on $S^{2}$, namely

$$
\begin{gathered}
A_{+}(\theta, \varphi)=s_{N}^{*}(\omega)(\theta, \varphi)=\left(j \circ s_{N}\right)^{*}(\tilde{\omega})(\theta, \varphi)=-\frac{i}{2}(1-\cos \theta) d \varphi, \\
A_{-}(\theta, \varphi)=s_{S}^{*}(\omega)(\theta, \varphi)=\left(j \circ s_{S}\right)^{*}(\tilde{\omega})(\theta, \varphi)=\frac{i}{2}(1+\cos \theta) d \varphi .
\end{gathered}
$$

The corresponding $u(1)$-valued 3 -vector potentials are

$$
\boldsymbol{A}_{+}=-i \frac{1-\cos \theta}{2 r \sin \theta} \hat{\varphi}, \boldsymbol{A}_{-}=+i \frac{1+\cos \theta}{2 r \sin \theta} \hat{\varphi},
$$

defined also at $\theta=0\left(\boldsymbol{A}_{+}\right)$and $\theta=\pi\left(\boldsymbol{A}_{-}\right)$:

$$
\boldsymbol{A}_{+}(\theta=0)=\boldsymbol{A}_{-}(\theta=\pi)=\mathbf{0}
$$

and on a 2 -sphere of arbitrary radius $r>0$. Clearly, the rotor of $\boldsymbol{A}_{+}$and $\boldsymbol{A}_{-}$gives the magnetic field $\boldsymbol{B}$.

The first Chern class of $\xi_{D}$ (taking $S^{2}$ with unit radius) is given by

$$
c_{1}\left(\xi_{D}\right)=\frac{i}{2 \pi}[F]
$$

where $[F]$ is the cohomology class of $F$ in $H^{2}\left(S^{2}\right)$ : cohomology of the 2-sphere in dimension 2 . The integral of $\frac{i}{2 \pi} F$ over $S^{2}$ gives the first Chern number of $\xi_{D}$ :

$$
\frac{i}{2 \pi} \int_{S^{2}} F=1
$$

This means that the magnetic charge is a measure of the topological non-triviality of the bundle $\xi_{D}$ i.e. of the space where it "lives". In other words, the monopole charge is not a property of the gauge field $A_{ \pm}$ itself, but of the $U(1)$-bundle on which the monopole is a connection.

\section{Bundle Morphism $\xi_{A-B} \rightarrow \xi_{D}$}

Using the homeomorphisms $\left(D_{0}^{2}\right)^{*} \cong \mathbb{C}^{*}$ and $S^{2} \cong \mathbb{C} \cup\{\infty\}$, it can be easily verified that $(\iota, \bar{\iota})$ given by

$$
\iota: \mathbb{C}^{*} \rightarrow \mathbb{C} \cup\{\infty\}, \iota(z)=z
$$

and

$$
\bar{\iota}: \mathbb{C}^{*} \times S^{1} \rightarrow S^{3}, \bar{\iota}\left(z, e^{i \varphi}\right)=\frac{(z, 1)}{\|(z, 1)\|} e^{i \varphi}
$$


with $\|(z, 1)\|=\sqrt{1+|z|^{2}}$, and $\left(\psi_{A-B}, \psi_{D}\right)$ the right actions

$$
\psi_{A-B}:\left(\mathbb{C}^{*} \times S^{1}\right) \times S^{1} \rightarrow \mathbb{C}^{*} \times S^{1}, \psi_{A-B}\left(\left(z, e^{i \alpha}\right), e^{i \beta}\right)=\left(z, e^{i(\alpha+\beta)}\right)
$$

and

$$
\psi_{D}: S^{3} \times S^{1} \rightarrow S^{3}, \psi_{D}\left(\left(z_{1}, z_{2}\right), e^{i \lambda}\right)=\left(z_{1} e^{i \lambda}, z_{2} e^{i \lambda}\right)
$$

is the unique bundle morphism

$$
\xi_{A-B} \rightarrow \xi_{D}
$$

induced by the inclusion $\iota$ i.e.

$$
\pi \circ \bar{\iota}=\iota \circ p r_{1}
$$

and

$$
\psi_{D} \circ\left(\bar{\iota} \times I d_{S^{1}}\right)=\bar{\iota} \circ \psi_{A-B}
$$

namely, with lower and upper parts of Diagram 1 commuting.

In fact:

$$
\begin{array}{ccc}
\left(\mathbb{C}^{*} \times S^{1}\right) \times S^{1} \stackrel{\bar{\iota} \times I d_{S^{1}}}{\longrightarrow} & S^{3} \times S^{1} \\
\psi_{A-B} \downarrow & & \downarrow \psi_{D} \\
\mathbb{C}^{*} \times S^{1} & \stackrel{\iota}{\longrightarrow} & S^{3} \\
p r_{1} \downarrow & & \downarrow \pi \\
\mathbb{C}^{*} & \stackrel{\iota}{\longrightarrow} & \mathbb{C} \cup\{\infty\}
\end{array}
$$

Diagram 1

$$
\begin{gathered}
\pi \circ \bar{\iota}\left(z, e^{i \varphi}\right)=\pi\left(\frac{(z, 1)}{\|(z, 1)\|} e^{i \varphi}\right)=z, \\
\iota \circ p r_{1}\left(z, e^{i \varphi}\right)=\iota(z)=z ; \\
\psi_{D} \circ\left(\bar{\iota} \times I d_{S^{1}}\right)\left(\left(z, e^{i \varphi}\right), e^{i \lambda}\right)=\psi_{D}\left(\bar{\iota}\left(z, e^{i \varphi}\right), e^{i \lambda}\right)=\frac{(z, 1)}{\|(z, 1)\|} e^{i(\varphi+\lambda)}, \\
\bar{\iota} \circ \psi_{A-B}\left(\left(z, e^{i \varphi}\right), e^{i \lambda}\right)=\bar{\iota}\left(z, e^{i(\varphi+\lambda)}\right)=\frac{(z, 1)}{\|(z, 1)\|} e^{i(\varphi+\lambda)} .
\end{gathered}
$$

\section{Pull-back of $\xi_{D}$ by $\iota: \iota^{*}\left(\xi_{D}\right)$}

The total space of the induced or pull-back bundle [14] of $\xi_{D}$ by $\iota, \iota^{*}\left(\xi_{D}\right): S^{1} \rightarrow P_{\iota^{*}\left(\xi_{D}\right)} \stackrel{p r_{1}}{\longrightarrow} \mathbb{C}^{*}$, is defined by

$$
P_{\iota^{*}\left(\xi_{D}\right)}=\left\{\left(z,\left(z_{1}, z_{2}\right)\right) \in \mathbb{C}^{*} \times S^{3}, \iota(z)=\pi\left(z_{1}, z_{2}\right)\right\}
$$

and must be such that both the upper and lower parts of Diagram 2 commute i.e. such that $\left(\iota, p r_{2}\right)$ is a bundle morphism $\iota^{*}\left(\xi_{D}\right) \rightarrow \xi_{D}$. In Diagram 2, $p r_{2}$ is the projection in the second entry, and

$$
\psi_{\iota^{*}\left(\xi_{D}\right)}: P_{\iota^{*}\left(\xi_{D}\right)} \times S^{1} \rightarrow P_{\iota^{*}\left(\xi_{D}\right)}, \psi_{\iota^{*}\left(\xi_{D}\right)}\left(\left(z,\left(z_{1}, z_{2}\right)\right), e^{i \lambda}\right)=\left(z,\left(z_{1}, z_{2}\right) e^{i \lambda}\right)
$$

is the right action of $S^{1}$ on $P_{\iota^{*}\left(\xi_{D}\right)}$.

$$
\begin{array}{ccc}
P_{\iota^{*}\left(\xi_{D}\right)} \times S^{1} & \stackrel{p r_{2} \times I d_{S^{1}}}{\longrightarrow} & S^{3} \times S^{1} \\
\psi_{\iota^{*}\left(\xi_{D}\right) \downarrow} & & \downarrow \psi_{D} \\
P_{\iota^{*}\left(\xi_{D}\right)} & \stackrel{p r_{2}}{\longrightarrow} & S^{3} \\
p r_{1} \downarrow & & \downarrow \pi \\
\mathbb{C}^{*} & \stackrel{\iota}{\longrightarrow} & \mathbb{C} \cup\{\infty\}
\end{array}
$$

Diagram 2 
From

$$
\iota \circ p r_{1}=\pi \circ p r_{2}
$$

one has:

$$
\begin{gathered}
\iota \circ p r_{1}\left(\left(z,\left(z_{1}, z_{2}\right)\right)=\iota(z)=z,\right. \\
\pi \circ p r_{2}\left(\left(z,\left(z_{1}, z_{2}\right)\right)=\pi\left(z_{1}, z_{2}\right)=z_{1} / z_{2},\right.
\end{gathered}
$$

so $z_{1}=z_{2} z$ and $\left\|\left(z_{1}, z_{2}\right)\right\|=1$ implies $\left(z_{1}, z_{2}\right)=\frac{(z, 1)}{\|(z, 1)\|} e^{i \varphi}$. Then,

$$
P_{\iota^{*}\left(\xi_{D}\right)}=\left\{\left(z, \frac{(z, 1)}{\|(z, 1)\|} e^{i \varphi}\right), z \in \mathbb{C}^{*}, \varphi \in[0,2 \pi)\right\} \subset \mathbb{C}^{*} \times S^{3} .
$$

On the other hand, it holds

$$
\psi_{D} \circ\left(p r_{2} \times I d_{S^{1}}\right)=p r_{2} \circ \psi_{\iota^{*}\left(\xi_{D}\right)} .
$$

In fact:

$$
\begin{gathered}
\psi_{D} \circ\left(p r_{2} \times I d_{S^{1}}\right)\left(\left(z,\left(z_{1}, z_{2}\right)\right), e^{i \lambda}\right)=\psi_{D}\left(\left(z_{1}, z_{2}\right) e^{i \lambda}\right)=\left(z_{1} e^{i \lambda}, z_{2} e^{i \lambda}\right), \\
p r_{2} \circ \psi_{\iota^{*}\left(\xi_{D}\right)}\left(\left(z,\left(z_{1}, z_{2}\right)\right), e^{i \lambda}\right)=p r_{2}\left(\left(z,\left(z_{1}, z_{2}\right) e^{i \lambda}\right)\right)=\left(z_{1}, z_{2}\right) e^{i \lambda}=\left(z_{1} e^{i \lambda}, z_{2} e^{i \lambda}\right) .
\end{gathered}
$$

\section{Bundle Isomorphism: $\iota^{*}\left(\xi_{D}\right) \stackrel{\cong}{\longrightarrow} \xi_{A-B}$}

In this section we exhibit a "natural" isomorphism between the $A-B$ bundle and the pull-back by the inclusion $\iota: \mathbb{C}^{*} \rightarrow \mathbb{C} \cup\{\infty\}$ (i.e. $\iota:\left(D_{0}^{2}\right)^{*} \rightarrow S^{2}$ up to homeomorphisms) of the Dirac bundle $\xi_{D}$ corresponding to unit magnetic charge, thus establishing a deep relation between the two systems $(A-B$ : experimentally observed, and $D$ : only theoretical, up to now).

The homeomorphism between the total spaces of the bundles is given by

$$
\Psi: P_{\iota^{*}\left(\xi_{D}\right)} \rightarrow \mathbb{C}^{*} \times S^{1}, \Psi\left(z, \frac{(z, 1)}{\|(z, 1)\|} e^{i \varphi}\right)=\left(z, e^{i \varphi}\right) .
$$

It is clear that $\Psi$ is continuous, one-to-one and onto, with continuous inverse $\Psi^{-1}$. It is easily verified that Diagram 3, corresponding to this isomorphism, commutes in its upper and lower parts i.e.

$$
p r_{1} \circ \Psi=I d_{\mathbb{C}^{*}} \circ p r_{1}
$$

and

$$
\begin{array}{ccc}
\psi_{A-B} \circ\left(\Psi \times I d_{S^{1}}\right)=\Psi \circ \psi_{\iota^{*}\left(\xi_{D}\right)} . \\
P_{\iota^{*}\left(\xi_{D}\right)} \times S^{1} \stackrel{\Psi \times I d_{S^{1}}}{\longrightarrow}\left(\mathbb{C}^{*} \times S^{1}\right) \times S^{1} \\
\psi_{\iota^{*}\left(\xi_{D}\right) \downarrow} & & \downarrow \psi_{A-B} \\
P_{\iota^{*}\left(\xi_{D}\right)} & \stackrel{\Psi}{\longrightarrow} & \mathbb{C}^{*} \times S^{1} \\
p r_{1} \downarrow & & \downarrow p r_{1} \\
\mathbb{C}^{*} & \stackrel{I d_{\mathbb{C}^{*}}^{\longrightarrow}}{\longrightarrow} & \mathbb{C}^{*} \\
& \text { Diagram 3 }
\end{array}
$$

In fact:

$$
\begin{aligned}
& p r_{1} \circ \Psi\left(z, \frac{(z, 1)}{\|(z, 1)\|} e^{i \varphi}\right)=p r_{1}\left(z, e^{i \varphi}\right)=z, \\
& I d_{\mathbb{C}^{*}} \circ p r_{1}\left(z, \frac{(z, 1)}{\|(z, 1)\|} e^{i \varphi}\right)=I d_{\mathbb{C}^{*}}(z)=z ; \\
& \psi_{A-B^{\circ}}\left(\Psi \times I d_{S^{1}}\right)\left(\left(z,\left(z_{1}, z_{2}\right)\right), e^{i \lambda}\right)=\psi_{A-B}\left(\Psi\left(\left(z,\left(z_{1}, z_{2}\right)\right), e^{i \lambda}\right)\right)=\Psi\left(z,\left(z_{1}, z_{2}\right)\right) e^{i \lambda}=\Psi\left(z, \frac{(z, 1)}{\|(z, 1)\|} e^{i \varphi}\right) e^{i \lambda} \\
& =\left(z, e^{i \varphi}\right) e^{i \lambda}=\left(z, e^{i(\varphi+\lambda)}\right), \\
& \Psi \circ \psi_{\iota^{*}\left(\xi_{D}\right)}\left(\left(z,\left(z_{1}, z_{2}\right)\right), e^{i \lambda}\right)=\Psi\left(z,\left(z_{1}, z_{2}\right) e^{i \lambda}\right)=\Psi\left(z, \frac{(z, 1)}{\|(z, 1)\|} e^{i(\varphi+\lambda)}\right)=\left(z, e^{i(\varphi+\lambda)}\right) .
\end{aligned}
$$




\section{Chern Classes}

$\xi_{A-B}$ is the pull-back of $\xi_{D}$ by the inclusion $\iota:\left(D_{0}^{2}\right)^{*} \rightarrow S^{2}$; however, since $\xi_{A-B}$ is trivial, then all its Chern classes must vanish. Then, in particular, we must verify the vanishing of the pull-back of $c_{1}$.

$\xi_{A-B}=\iota^{*}\left(\xi_{D}\right)$ passes to cohomology [15] in the form

$$
\iota^{*}: H^{*}\left(S^{2}\right) \rightarrow H^{*}\left(\left(D_{0}^{2}\right)^{*}\right)
$$

i.e.

$$
\iota^{*}: H^{k}\left(S^{2}\right) \rightarrow H^{k}\left(\left(D_{0}^{2}\right)^{*}\right), k=0,1,2
$$

where

$$
H^{*}\left(S^{2}\right)=\left(H^{0}\left(S^{2}\right), H^{1}\left(S^{2}\right), H^{2}\left(S^{2}\right)\right) \cong(\mathbb{R}, 0, \mathbb{R})
$$

and

$$
H^{*}\left(\left(D_{0}^{2}\right)^{*}\right)=\left(H^{0}\left(\left(D_{0}^{2}\right)^{*}\right), H^{1}\left(\left(D_{0}^{2}\right)^{*}\right), H^{2}\left(\left(D_{0}^{2}\right)^{*}\right)\right) \cong(\mathbb{R}, \mathbb{R}, 0)
$$

are the cohomology groups of the 2 -sphere and the punctured disk respectively. $H^{*}\left(\left(D_{0}^{2}\right)^{*}\right) \cong H^{*}\left(S^{1}\right)$ by homotopy invariance. Since $c_{1} \in H^{2}\left(S^{2}\right)$, then

$$
\iota^{*}\left(c_{1}\right)=0 .
$$

\section{Pull-back of the Dirac Connection and Vanishing of the $A-B$ Effect}

In terms of the cartesian coordinates in $\mathbb{R}^{3},(x, y, z)=r(\sin \theta \cos \varphi, \sin \theta \sin \varphi, \cos \theta)$ with $\theta \in(0, \pi)$ and $\varphi \in[0,2 \pi)$ which implies $(x, y, z) \neq(0,0, z)$, the monopole potentials $A_{ \pm}$of equations (16a) and (16b) are given by

$$
A_{ \pm}(x, y, z)=\left(A_{ \pm}\right)_{x} d x+\left(A_{ \pm}\right)_{y} d y
$$

with

$$
\left(A_{ \pm}\right)_{x}(x, y, z)= \pm \frac{i}{2}\left(\frac{y}{x^{2}+y^{2}}\right)\left(1 \mp \frac{z}{\sqrt{x^{2}+y^{2}+z^{2}}}\right),\left(A_{ \pm}\right)_{y}(x, y, z)=\mp \frac{i}{2}\left(\frac{x}{x^{2}+y^{2}}\right)\left(1 \mp \frac{z}{\sqrt{x^{2}+y^{2}+z^{2}}}\right) .
$$

(Notice that $\left[\left(A_{ \pm}\right)_{x}\right]=\left[\left(A_{ \pm}\right)_{y}\right]=[L]^{-1}$ since $[x]=[y]=[z]=[L]$ while $\left[A_{ \pm}\right]=[L]^{0}$, $L$ : length.)

To pull-back by $\iota$ these 1 -forms to $\left(D_{0}^{2}\right)^{*}$ we must first restrict $A_{ \pm}$to $z=0$ and then perform the pull-back operation, which reduces to the identity:

$$
\iota^{*}\left(A_{ \pm}(x, y, 0)\right)= \pm \frac{i}{2}\left(\frac{y d x-x d y}{x^{2}+y^{2}}\right):=i a_{ \pm}(x, y)
$$

with

$$
a_{ \pm}(x, y)=\mp \frac{1}{2}\left(\frac{x d y-y d x}{x^{2}+y^{2}}\right)
$$

the real-valued $A-B$ potential 1-forms. Clearly, $a_{ \pm}$are closed $\left(d a_{ \pm}=0\right)$ but not exact since $a_{ \pm}=\mp \frac{1}{2} d \varphi$ only for $\varphi \in(0,2 \pi)$. If we surround the thin solenoid in the $A-B$ side with closed curves $\gamma_{ \pm}$with $\gamma_{-}=-\gamma_{+}$, then the surrounded magnetic flux is

$\Phi_{A-B}=\int_{\gamma_{+}} a_{+}+\int_{\gamma_{-}} a_{-}=\int_{\gamma_{+}} a_{+}+\int_{\gamma_{-}}\left(-a_{+}\right)=\int_{\gamma_{+}} a_{+}-\int_{\gamma_{+}}\left(-a_{+}\right)=2 \int_{\gamma_{+}} a_{+}=2 \int_{\gamma_{+}}\left(-\frac{1}{2} d \varphi\right)=-2 \pi$,

which coincides, up to a sign, with the flux of the monopole:

$$
\Phi_{D}=\int_{S^{2}} \boldsymbol{B}=\left(\frac{1}{2}\right) \int_{S^{2}} \frac{\hat{r} \cdot \hat{r}}{r^{2}}=\left(\frac{1}{2}\right) 4 \pi=2 \pi .
$$

But this implies that the $A-B$ effect vanishes if and only if the value of the electric charge $|e|$ is an integer: the D.Q.C. for the present case where $g=\frac{1}{2}$. In fact, with $\Phi_{0}=\frac{2 \pi}{|e|}$ the quantum of magnetic flux associated with the charge $|e|$, the phase change of the wave function in the $A-B$ experiment due to the presence of magnetic flux is

$$
e^{-i|e| \Phi_{A-B}}=e^{-2 \pi i \frac{\Phi_{A-B}}{\Phi_{0}}}=e^{2 \pi i \frac{\Phi_{D}}{\Phi_{0}}}=e^{i|e|\left(\frac{1}{2}\right) 4 \pi}=e^{2 \pi i|e|}=1 \Leftrightarrow|e|=n \in \mathbb{Z} .
$$

(For arbitrary $g$, the D.Q.C. would be $|e| g=\frac{n}{2}$.) 


\section{Final Comments}

It is well known that the $A-B$ effect and the Dirac monopole are closed related [16]; in particular the disappearance of the Dirac string simultaneously with the vanishing of the $A-B$ effect when appropriate conditions of the magnetic fluxes are fulfilled [17]. In the present paper, the above relation has been described in the context of the fiber bundles associated with both phenomena, respectively $\xi_{A-B}$ (trivial) and $\xi_{D}$ (non-trivial Hopf bundle). The remarkable fact is that $\xi_{A-B}$ turns out to be the pull-back of $\xi_{D}$ by the inclusion $\iota$ of the corresponding base spaces, which allows to discuss the above relation in a purely geometric language. It would be interesting to investigate if this bundle theoretic relation exists in non-abelian cases.

Acknowledgments. The author thanks Gregory L. Naber and Sebastián Nájera Valencia for useful and enlightening discussions.

\section{References}

1. Y. Aharonov, D. Bohm, Physical Review 15, 485 (1959).

2. P.A.M. Dirac, Proceedings of the Royal Society A133, 60 (1931).

3. P.A.M. Dirac, Physical Review 74, 817 (1948).

4. R.G. Chambers, Physical Review Letters 5, 3 (1960).

5. M. Peshkin, A. Tonomura, The Aharonov-Bohm Effect, (Springer, Berlin, 1989).

6. J. Preskill, Annual Review of Nuclear and Particle Science 34, 451 (1984).

7. J. Polchinski, International Journal of Modern Physics A19, 145 (2004).

8. M. Aguilar, M. Socolovsky, International Journal of Theoretical Physics 41, 839 (2002).

9. M. Socolovsky, Encyclopedia of Mathematical Physics, eds. J.P. Francoise, G.L. Naber, T.S. Sun; Elsevier; 191 (2006).

10. G.L. Naber, Topology, Geometry, and Gauge Fields. Foundations, (Springer-Verlag, N.Y., 1997).

11. N. Steenrod, The Topology of Fibre Bundles, (Princeton University Press, N.J., 1951).

12. M. Socolovsky, Aportaciones Matemáticas, Notas de Investigación (Sociedad Matemática Mexicana) 6, 141 (1992).

13. H. Hopf, Mathematische Annalen 104, 637 (1931).

14. A. Trautman, International Journal of Theoretical Physics 16, 561 (1977).

15. D. Husemoller, Basic Bundle Theory and K-Cohomology Invariants, Lecture Notes in Physics 726, (Springer, Berlin Heidelberg, 2008).

16. T.T. Wu, C.N. Yang, Physical Review D 12, 3845 (1975).

17. A. Rajantie, Contemporary Physics 53, 195 (2012). 